\title{
Connecting Moduli Spaces of Calabi-Yau Threefolds
}

\author{
Paul S. Green ${ }^{1}$ and Tristan Hübsch ${ }^{2 \star \star \star \star}$ \\ ${ }^{1}$ Department of Mathematics, University of Maryland, College Park, MD 20742, USA \\ 2 Theory Group, Department of Physics, University of Texas, Austin, TX 78712, USA
}

\begin{abstract}
We demonstrate that many families of Calabi-Yau threefolds consist generically of small resolutions of nodal forms in other families and, in fact, that a large class of families is connected by this relation. Our result resonates with a conjecture of Reid that Calabi-Yau threefolds may have a universal moduli space even though they are of different homotopy types. Such ideas tie quite naturally to alluring prospects of unifying (super)string models.
\end{abstract}

\section{Introduction}

There is a growing list of constructions of Calabi-Yau threefolds, i.e., compact complex threefolds with vanishing first Chern class. Among them, there exists a large family of non-singular Calabi-Yau threefolds embedded as complete intersections in products of complex projective spaces (CICYs) which were studied by the authors and others $[1,2]$. A particularly interesting phenomenon was discussed in ref. [2], where it was called "contracting" (vs. its inverse, "splitting") whereby the non-singular varieties of one family of CICYs turn out generically to be small resolutions of nodal varieties in another family. Here we make a suitable generalization of this notion and study its effect on the Calabi-Yau threefolds $\mathscr{M}$ together with their deformation spaces $\Theta$.

Clemens [3] has studied "double solids", i.e., double covers of $\mathbb{P}^{3}$ branched over possibly singular surfaces; in the case when the branching surface is octic and nodal, a small resolution of the double cover is a Calabi-Yau manifold. We show that in several cases CICYs are small resolutions of double covers of $\mathbb{P}^{3}$ branched over nodal octics, and prove similar results for the generalization of double solids to branched double covers of more general threefolds.

Reid has suggested [4] that if $\Theta^{\prime}$ contracts to $\Theta$ (as will be specified below), it is reasonable to identify $\Theta^{\prime}$ with a singular stratum of $\Theta$, and conjectures the existence of a unified moduli space of (generically non-algebraic) threefolds of

\footnotetext{
* Supported by the Robert A. Welch Foundation and NSF Grants: PHY 8503890 and PHY 8605978

$\star \star$ On leave from "Ruder Bošković" Institute, Bijenička 54, YU-41000 Zagreb, Croatia, Yugoslavia
} 
which moduli spaces of many of the known Calabi-Yau threefolds may be regarded as strata.

In support of this conjecture, we present here a large collection of deformation spaces of Calabi-Yau threefolds of which we can prove that it is connected by the relation of contraction in a sense that we shall make precise.

The plan of the paper is as follows: We give necessary definitions and set up the framework of our analysis in Sect. 2. In Sect. 3, we review the constructions of threefolds as complete intersections in products of complex projective spaces and review the phenomenon of "splitting" as discussed in ref. [2]. In Sect. 4, we discuss a generalization/specialization of the class of double solids studied by Clemens [3]. We study the phenomenon of contraction among the threefolds of this class as well as contractions which relate these threefolds to those studied in the previous section. In Sect. 5, we show that all the deformation spaces studied in this paper can be connected by the relation of contraction. Finally in Sect. 6, we discuss the implications of this phenomenon to physics applications. This ties quite naturally to some alluring prospects for unification of (super)string models.

\section{Holomorphic Families and Contractions}

An holomorphic family of compact projective $n$-folds is a triple $(\Theta, \mathbb{E}, \varpi)$, where $\Theta$ and $\mathbb{E}$ are complex manifolds and $\varpi: \mathbb{E} \rightarrow \Theta$ is a holomorphic map whose fibres $\mathscr{M}$, said to belong to the family, are compact projective varieties and whose generic fibre is non-singular of dimension $n$. ( $\Theta$ is a deformation space for $\mathscr{M}$.)

A holomorphic map $\wp: \mathbb{E}^{\prime} \rightarrow \mathbb{E}$ is called a contraction of $\left(\Theta^{\prime}, \mathbb{E}^{\prime}, \varpi^{\prime}\right)$ to $(\Theta, \mathbb{E}, \varpi)$ if

i. $\wp$ is a fibrewise map and maps each fibre $\mathscr{M}^{\prime}$ of $\varpi^{\prime}$ onto a fibre $\mathscr{M}$ of $\varpi$.

ii. The restriction of $\wp$ to a generic fibre $\mathscr{M}^{\prime}$ of $\varpi^{\prime}$ is a resolution of the variety $\wp\left(\mathscr{M}^{\prime}\right)$.

We shall write $\Theta$ for $(\Theta, \mathbb{E}, \varpi)$, where there is no danger of confusion, and we shall say $\Theta$ contracts to $\Theta^{\prime}$ if there is a contraction from $(\Theta, \mathbb{E}, \varpi)$ to $\left(\Theta^{\prime}, \mathbb{E}^{\prime}, \varpi^{\prime}\right)$.

In case $\Theta$ and $\Theta^{\prime}$ are families of thresholds, we say $\wp$ is a contraction by small resolutions if the following stronger form of ii. holds:

ii'. The restriction of $\wp$ to a generic fibre $\mathscr{M}$ of $\varpi$ is a small resolution of the nodal variety $\wp(\mathscr{M}){ }^{1}$

Let $(\Theta, \mathbb{E}, \varpi)$ be an holomorphic family, $\mathscr{L}$ a line bundle over $\mathbb{E}$, and $\Lambda$ a linear space of global sections of $\mathscr{L}^{2}$ such that $\Lambda$ generates $\mathscr{L}^{2}$ (we are leaving open the possibility that $\Lambda$ is not the full space of sections of $\mathscr{L}^{2}$ ). We define an induced double covering family $\left({ }_{\Lambda} \Theta,{ }_{\Lambda} \mathbb{E},{ }_{\Lambda} \varpi\right)$ as follows: Let $h_{\Lambda}$ be the hyperplane bundle of $\mathbb{P}(\Lambda)$, the projective space of the linear space $\Lambda$. There is a natural section $\eta$ of $\mathscr{L}^{2} \otimes h_{\Lambda}$ over $\mathbb{E} \times \mathbb{P}(\Lambda)$ defined by projectivising the map $(e, \lambda) \rightarrow \lambda(e)$, where $e \in \mathbb{E}$ and $\lambda \in \Lambda$. ${ }_{\Lambda} \mathbb{E}$ is the double cover of $\mathbb{E} \times \mathbb{P}(\Lambda)$ branched over the 0 -locus of $\eta$. Note that $\eta$ has a nowhere vanishing one-jet, so that ${ }_{\Lambda} \mathbb{E}$ is again a complex manifold. ${ }_{\Lambda} \Theta$ is $\Theta \otimes \mathbb{P}(\Lambda)$. The map ${ }_{\Lambda} \varpi$ is the double covering map followed by the projection on $\Theta \otimes \mathbb{P}(\Lambda)$.

\footnotetext{
${ }^{1}$ See ref. [5] for the definition of a nodal variety and ref. [6] for the definition of a small resolution
} 
If $\left(\Theta^{\prime}, \mathbb{E}^{\prime}, \varpi^{\prime}\right)$ is another holomorphic family, $\wp: \mathbb{E}^{\prime} \rightarrow \mathbb{E}$ is a contraction, and $\mathscr{L}^{\prime}=\wp^{*}(\mathscr{L})$ is the pull-back of $\mathscr{L}$ by $\wp$ and $\wp^{*}: \Lambda \rightarrow \Gamma\left(\mathscr{L}^{\prime}\right)$ is a monomorphism with image $\Lambda^{\prime}$, we define

$$
\wp_{\Lambda}:{ }_{\Lambda} \mathbb{E}^{\prime} \rightarrow{ }_{\Lambda} \mathbb{E}
$$

by $\wp_{\Lambda}\left(e^{\prime}, \wp^{*}(\lambda)\right)=(\wp(e), \lambda)$, with $e^{\prime} \in \mathbb{E}^{\prime}$ and $\lambda \in \Lambda$.

The definitions and the notation have been framed to lead to a straightforward proof of

Proposition 1. $\wp_{\Lambda}$ is a contraction of holomorphic families from $\left({ }_{\Lambda^{\prime}} \Theta^{\prime},{ }_{\Lambda^{\prime}} \mathbb{E}^{\prime},{ }_{\Lambda^{\prime}} \varpi^{\prime}\right)$ to $\left({ }_{\Lambda} \Theta,{ }_{\Lambda} \mathbb{E},{ }_{\Lambda}^{\varpi)}\right.$. Moreover, if $\wp$ is a contraction by small resolution, so is ${ }_{\Lambda}$.

\section{Complete Intersections in Products of Projective Spaces}

A configuration is a pair $[\mathscr{W} \| \mathscr{E}]$, where $\mathscr{W}$ is a non-singular projective variety and $\mathscr{E}$ is a sequence of line bundles $\left(\mathscr{E}_{1}, \ldots, \mathscr{E}_{K}\right)$ each of which is generated by global sections.

We define the holomorphic family $\left(\Theta_{\mathscr{E}}, \mathbb{E}_{\mathscr{E}}, \varpi_{\mathscr{E}}\right)$ of the configuration $[\mathscr{W} \| \mathscr{E}]$ by setting

$$
\Theta=\mathbb{P}\left(\Gamma\left(\mathscr{E}_{1}\right)\right) \times \cdots \times \mathbb{P}\left(\Gamma\left(\mathscr{E}_{K}\right)\right),
$$

and defining $\mathbb{E}$ to be the subset of $\Theta \times \mathscr{W}$ for which each component of the $\Theta$ coordinate vanishes on the $\mathscr{W}$ coordinate. $\varpi_{\mathscr{E}}$ is the projection on $\Theta$. Here $\Gamma(\quad)$ denotes the space of global sections. Note that the generic fibre $\mathscr{M}$ of $\varpi$ is a non-singular intersection of $K$ hypersurfaces in $\mathscr{W}$. For any fibre $\mathscr{M}$ of $\varpi$ we shall write $\mathscr{M} \in[\mathscr{W} \| \mathscr{E}]$ and call $\mathscr{M}$ a member of the configuration.

It should be pointed out that the deformation spaces $\Theta$ used here are considerably redundant. This is the natural context in which to formulate the constructions we are investigating; a formulation in terms of effective deformation spaces (moduli spaces) could probably be contrived but we do not believe that the benefits to be derived therefrom would compensate for the considerable loss of transparency.

The case when

$$
\mathscr{W}=\mathbb{P}_{1}^{n_{1}} \times \cdots \times \mathbb{P}_{m}^{n_{m}} \text { and } \mathscr{E}_{a}=\bigotimes_{r=1}^{m}\left(h_{r}\right)^{q_{a}^{r}},
$$

where $h_{r}$ denotes the hyperplane bundle of $\mathbb{P}_{r}^{n_{r}}$ has been studied in ref. $[1,2]$. In our notation, $\mathscr{W}$ in such a case is represented by the $m$-dimensional positive integer valued column vector $\mathbf{n}=\left[n_{r}\right]$ and $\mathscr{E}$ is represented by an $m \times K$-dimensional non-negative integer valued matrix $\mathbf{a}=\left[q_{a}^{r}\right], r=1, \ldots, m$ and $a=1, \ldots, K$. This defines the configuration matrix $[\mathbf{n} \| \mathbf{q}]$. Configuration matrices which differ only by permutations of the $m$ rows of $[\mathbf{n} \| \mathbf{q}]$ and/or permutations of the $K$ columns of $\mathbf{q}$ are for all practical purposes equivalent and we will not distinguish among them. Also, we shall often not distinguish notationally between a configuration matrix and the corresponding configuration, and will write, for instance, $\mathscr{M} \in[\mathbf{n} \| \mathbf{q}]$.

We have been primarily interested in configuration matrices the generic variety 
$\mathscr{M}$ of which is a Calabi-Yau threefold. This requires that

$$
\sum_{a=1}^{K} q_{a}^{r}=n_{r}+1, \quad \forall r
$$

and we adopt the acronym of ref. [2] CICY (for complete intersection Calabi-Yau) for manifolds belonging to such configurations. For auxilliary purposes, however, we also consider configuration matrices satisfying the weaker condition

$$
\sum_{a=1}^{K} q_{a}^{r} \leqq n_{r}+1, \quad \forall r
$$

A variety $\mathscr{M}$ belonging to such a configuration, provided the inequality is strict for at least one $r$, has the property that its anti-canonical bundle, $K^{*}{ }_{\mathscr{M}}$, is non-trivial and generated by global sections. We shall refer to such varieties as almost Fano ${ }^{2}$ complete intersections (AFCI for short).

It suffices to consider configuration matrices that satisfy:

$$
\sum_{r=1}^{m} q_{a}^{r} \geqq 2, \quad \forall a,
$$

since hyperplane sections of only one factor $\mathbb{P}^{n}$ in $\mathscr{W}$ simply reduce this $\mathbb{P}^{n}$ to $\mathbb{p}^{n-1}$

We now describe a process that was called "contracting" in ref. [2]. To that end consider a generic non-singular manifold

$$
\mathscr{M} \in\left[\mathbb{P}^{n} \times \mathscr{W} \| \mathscr{E}\right], \quad \mathscr{E}=\left(h \otimes \mathscr{F}_{1}\right), \ldots,\left(h \otimes \mathscr{F}_{n+1}\right),
$$

where $\mathscr{W}$ represents a AFCI fourfold. Notice that $\mathscr{M}$ is a Calabi-Yau threefold if $\mathscr{F}_{K}:=\otimes_{f=1}^{n+1} \mathscr{F}_{f}$ is the anti-canonical bundle of $\mathscr{W}$, denoted $K^{*}{ }_{\mathscr{W}}$.

Writing $\left(z_{0}, \ldots, z_{n}\right)$ for the homogeneous coordinates of $\mathbb{P}^{n}$ (and thus a basis for the space of sections of $h)$ and $w \in \mathscr{W}, \mathscr{M}$ is the locus of $(n+1)$ equations of the form:

$$
\sum_{i=0}^{n} \phi_{f}^{i}(w) z_{i}=0, \quad f=1, \ldots, n+1,
$$

where each $\phi_{f}{ }^{i}$ is a section of $\mathscr{F}_{f}$. Since $z_{i}$ cannot all vanish simultaneously, Eq. (3.4) implies that

$$
\Delta(w):=\operatorname{det}\left[\phi_{f}^{i}(w)\right]=0 .
$$

$\Delta$ is clearly a (non-generic) section of the bundle $\mathscr{F}_{K}$.

We write $\pi:\left(\mathscr{W} \times \mathbb{P}^{n}\right) \rightarrow \mathscr{W}$ for the projection.

Theorem $1 \pi(\mathscr{M})$ belongs to the configuration,

$$
\left[\mathscr{W} \| \mathscr{F}_{K}\right],
$$

and, for a generic choice of $\mathscr{M}, \pi(\mathscr{M})$ has no singularities other than nodes.

\footnotetext{
${ }^{2}$ This is derived from the use of the term "Fano threefolds" for threefolds with ample anti-canonical sheaf
} 
A proof of this theorem is outlined in ref. [2] (Sect. III). It starts with the observation that $\Delta$ is singular precisely when the matrix of sections, (3.4) has corank 2 . It is then observed that this requirement already imposes 4 constraints, and therefore occurs generically only at points. Furthermore, the vanishing of the Hessian imposes still another constraint and therefore generically does not happen. This argument is essentially correct on a philosophical level and entirely satisfactory for $n=1$. A completely rigorous proof for all values of $n$ can be constructed by the systematic use of Bertini's theorem and the theorem of Grauert and Artin $[7,6]$ to the effect that a rational curve in a threefold, along which the canonical bundle of the threefold is trivial and the normal bundle of which does not admit a section, can be blown down to a node. The details of such a proof do not come within the scope of a note such as the present one and will be given in a forthcoming paper [8]. These remarks should be understood to apply also to the proof of Lemma 1 in the next section.

Corollary 1. The determinant map det: $\left[\phi_{f}{ }^{i}\right] \rightarrow \Delta$ induces a contraction by small resolutions

$$
\wp:\left(\Theta_{\mathscr{E}}, \mathbb{E}_{\mathscr{E}}, \varpi_{\mathscr{E}}\right)=\left(\Theta_{\mathscr{F}_{K}}, \mathbb{E}_{\mathscr{F}_{K}}, \varpi_{\mathscr{F}_{K}}\right) .
$$

Proof. Using the above notation, we have in the case of the configuration (3.3) coordinates $\left(w, p ;\left[\phi_{f}^{i}\right]\right)$ for $\mathscr{W} \times \mathbb{P}^{n}$ and $\Theta_{\mathscr{E}} .\left(w ; \theta_{K}\right)$ are coordinates of $\mathscr{W}$ and $\Theta_{\mathscr{F}_{K}}$ for the configuration (3.6). The contraction map $\wp$ is now defined by

$$
\wp\left(w, p ; \phi_{f}^{i}\right) \rightarrow(w ; \Delta) .
$$

The verification of criteria i. and ii.' is now straightforward.

Remark. In the alternate notation for configurations mentioned above, the contraction acts

$$
\begin{array}{r}
\wp: \mathscr{M} \in\left[\begin{array}{c||cccccc}
n_{1} & q_{1}^{1} & \cdots & q_{K}^{1} & \bar{q}_{1}^{1} & \cdots & \bar{q}_{n}^{1} \\
\vdots & \vdots & \ddots & \vdots & \vdots & \ddots & \vdots \\
n_{m} & q_{1}^{m} & \cdots & q_{K}^{m} & \bar{q}_{1}^{m} & \cdots & \bar{q}_{n}^{m} \\
n & 0 & \cdots & 0 & 1 & \cdots & 1
\end{array}\right] \\
\rightarrow \wp(\mathscr{M}) \in\left[\begin{array}{c||ccccc}
n_{1} & q_{1}^{1} & \cdots & q_{K}^{1} & \left(\bar{q}_{1}^{1}+\cdots+\bar{q}_{n}^{1}\right) \\
\vdots & \vdots & \ddots & \vdots & \vdots & \\
n_{m} & q_{1}^{m} & \cdots & q_{K}^{m} & \left(\bar{q}_{1}^{m}+\cdots+\bar{q}_{n}^{m}\right)
\end{array}\right],
\end{array}
$$

where $\bar{q}_{f}^{i}=\operatorname{deg} \phi_{f}{ }^{i}$, and where the AFCI $\mathscr{W}$ is a generic member of the configuration

$$
\left[\begin{array}{c||ccc}
n_{1} & q_{1}^{1} & \cdots & q_{K}^{1} \\
\vdots & \vdots & \ddots & \vdots \\
n_{m} & q_{1}^{m} & \cdots & q_{K}^{m}
\end{array}\right]
$$

\section{Calabi-Yau Double Solids}

Clemens has studied two-fold coverings of $\mathbb{P}^{3}$ branched over possibly singular 
hyper-surfaces. In the case when the hypersurface has degree eight and is non-singular, the two-fold cover is a Calabi-Yau threefold.

4.1. Double Covered Almost Fano Threefolds. The constructions of Clemens has a straightforward generalization, replacing $\mathbb{P}^{3}$ with an AFCI threefold (as defined in Sect. 3). To relate the canonical class of the AFCI with the canonical class of its double cover we shall need a standard result:

Proposition 2. Let $\mathscr{V}$ and $\tilde{\mathscr{V}}$ be two $n$-folds and $\pi: \tilde{\mathscr{V}} \rightarrow \mathscr{V}$ be a double cover branched over the non-singular 0-locus of a section $\eta$ of $\mathscr{L}^{2}$, where $\mathscr{L}$ is a line bundle over $\mathscr{V}$. Then

$$
K^{*}{ }_{\tilde{\gamma}}=\pi^{*}\left(K^{*}{ }_{r} \otimes \mathscr{L}^{*}\right) .
$$

Corollary 2. The double cover of an AFCI branched over the 0-locus of a section of the square of the anti-canonical bundle is a Calabi-Yau threefold.

4.2 Linking Calabi-Yau Double Solids with CICYs. Now we relate the Calabi-Yau threefolds defined in Corollary 2 with the constructions reviewed in Sect. 3.

Proposition 3. A (generic) manifold

$$
\mathscr{M} \in\left[\begin{array}{l|l}
3 & 4 \\
1 & 2
\end{array}\right]
$$

is a small resolution of a twofold covering of $\mathbb{P}^{3}$, branched over an octic surface with 64 nodes.

Proof. Let $(x, y)$ be homogeneous coordinates of $\mathbb{P}^{1}$. The constraint equation defining $\mathscr{M}$ is

$$
A x^{2}+B x y+C y^{2}=0,
$$

where $A, B$ and $C$ are quartics over $\mathbb{P}^{3}$. Let $\pi$ be the projection of $\mathscr{M}$ on $\mathbb{P}^{3}$, and $\Delta=B^{2}-4 A C$. For $p$ a point in $\mathbb{P}^{3}, \pi^{-1}(p)$ consists of

1. Two points if $\Delta(p) \neq 0$;

2. A point if $\Delta=0$, but at least one of $A(p), B(p)$ and $C(p)$ does not vanish;

3. A copy of $\mathbb{P}^{1}$, if $A(p)=B(p)=C(p)=0$.

For a generic $\mathscr{M}$, condition 3 . will hold at 64 points at each of which the three quadrics in $\mathbb{P}^{3}$ intersect transversally. This suffices to show as in ref. [2] (Sect. III) that these points are nodes of the otherwise smooth surface $\Delta=0$ in $\mathbb{P}^{3} . \mathscr{M}$ is evidently a small resolution of the double cover of $\mathbb{P}^{3}$, branched over the surface $\Delta=0$.

This correspondence between CICYs and double solids can be generalized in two straightforward ways.

To start with, consider a generic manifold

$$
\mathscr{M} \in\left[\begin{array}{l||ll}
3 & a & b \\
2 & 2 & 1
\end{array}\right],
$$


the defining equations of which are

$$
\begin{aligned}
\sum_{i, j=0}^{2} Q^{i j} z_{i} z_{j} & =0, \\
\sum_{i=0}^{2} L^{i} z_{i} & =0,
\end{aligned}
$$

with $\left(z_{0}, z_{1}, z_{2}\right) \in \mathbb{P}^{2}, Q^{i j}$ and $L^{i}$ are homogeneous in coordinates of $\mathbb{P}^{3}$ and of degree $a$ and $b$ respectively. Let $\pi$ again be the projection of $\mathscr{M}$ on $\mathbb{P}^{3}$. For $p$ a point in $\mathbb{P}^{3}, \pi^{-1}(p)$ is the intersection of the quadric (4.1) and the line (4.2), and consists of

1. Two points when the line (4.2) is transverse to the quadric (4.1) at regular points;

2. A point when the line (4.2) is tangent to the quadric (4.1) or meets it transversely at a double point;

3. A copy of $\mathbb{P}^{1}$ when

(a) $L^{i}(p)=0$ and the quadric $(4.1)$ is non-degenerate. Here $\mathbb{P}^{1}$ is identified with the quadric (4.1) in $\mathbb{P}^{3}$;

(b) The quadric (4.1) degenerates in a union of two lines at least one of which coincides with the line (4.2).

Both the cases $Q^{i j}(p)=0, \forall i, j$ and the case $L^{1}(p)=L^{2}(p)=L^{3}(p)=0$ simultaneously with the degeneration of the quadric (4.1) are precluded by the genericity of the choice of $\mathscr{M}$.

The above analysis generalizes straightforwardly when $\mathbb{P}^{3}$ is replaced with a non-singular AFCI $\mathscr{W}$ as defined in the previous section. There exist further generalizations along this line, for which we prove

Lemma 1. A generic Calabi-Yan manifold

$$
\mathscr{M} \in\left[\mathscr{W} \times \mathbb{P}^{n} \|\left(\mathscr{A} \otimes h^{2}\right),\left(\mathscr{B}_{2} \otimes h\right), \ldots,\left(\mathscr{B}_{n} \otimes h\right)\right]
$$

is a small resolution of the double cover of $\mathscr{W}$ branched over the 0-locus of a section of the square of the anti-canonical bundle $K^{*}$ (which is the product $\mathscr{A} \otimes \mathscr{B}_{2} \otimes \cdots \otimes \mathscr{B}_{n}$ ). The composition $\pi$ of the resolution with the covering is given by the projection on $\mathscr{W}$.

Proof. Denoting by $\left(z_{0}, \ldots, z_{n}\right)$ the homogeneous coordinates of $\mathbb{P}^{n}$ and for $w \in \mathscr{W}$, the defining equations of $\mathscr{M}$ may be written as

$$
\begin{aligned}
\sum_{i, j=0}^{n} A^{i j}(w) z_{i} z_{j} & =0, \\
\sum_{i=0}^{n} B_{f}^{i}(w) z_{i} & =0, \quad f=2, \ldots, n .
\end{aligned}
$$

For each $w \in \mathscr{W}$, let $\mathscr{Z}_{w}$ be the projectivised locus of Eq. (4.4) and let $\mathbf{Q}_{w}$ be the restriction of the quadratic relation (4.3) to $\mathscr{Z}_{w}$. We write $d_{w}$ for the dimension of $\mathscr{Z}_{w}$ and $c_{w}$ for the corank of $\mathbf{Q}_{w}$. For fixed $w$, the set of possible choices of the coefficient sections in (4.4) for which the $i^{\text {th }}$ equation is linearly dependent on the previous $i-1$ has codimension $n+1-i$. Thus the set of possible choices of the coefficient sections for which this is the case for any $w$ has codimension $n-i-2$ 
since $\mathscr{W}$ has dimension 3 . It follows that for generic choice of $\mathscr{M}$ the first $n-2$ of the $n-1$ equations (4.4) are linearly independent for all $w$ so that the possible values for $d_{w}$ are 1 and 2 .

A similar analysis shows for $d_{w}=1$ the possible values for $c_{w}$ are 0,1 and 2 , while for $d_{w}=2,0$ is the only possible value for $c_{w}$. We are now ready to consider the possibilities for $\pi^{-1}(w)$ for a generic choice of $\mathscr{M}$.

$d_{w}=1 ; c_{w}=0$ : This is the generic case and $\pi^{-1}(w)$ consists of two points corresponding to the two distinct solutions of $\mathbf{Q}_{w}$.

$d_{w}=1 ; c_{w}=1: \pi^{-1}(w)$ consists of a single point corresponding to the unique solution to $\mathbf{Q}_{w}$. This occurs at the regular points of a hypersurface which is the null locus of

$$
A^{i_{0} j_{0}} \otimes A^{i_{1} j_{1}} \otimes B_{2}^{i_{2}} \otimes B_{2}^{j_{2}} \otimes \cdots \otimes B_{n}^{i_{n}} \otimes B_{n}^{j_{n}} \varepsilon_{i_{0} \cdots i_{n}} \varepsilon_{j_{0} \cdots j_{n}},
$$

and whose singularities are given by the next two cases:

$\mathrm{d}_{w}=2 ; c_{w}=0: p^{-1}(w)$ is a smooth quadric on $\mathscr{Z}_{w} \cong \mathbb{P}^{2}$, which is abstractly a copy of $\mathbb{P}^{1}$.

$d_{w}=1 ; c_{w}=2: \mathbf{Q}_{w}$ is vacuous and $\pi^{-1}(w)$ consists of $\mathscr{Z}_{w}$, again a copy of $\mathbb{P}^{1}$.

By a careful analysis which we shall not reproduce here, (see, however, the discussion following Theorem 1) it can be shown that the singularities of Eq. (4.5), corresponding to the last two cases, are nodes. If the $\mathbb{P}^{1} \mathrm{~s}$ lying over the nodes are collapsed, we obtain the double cover of $\mathscr{W}$ branched along the zero locus of Eq. (4.5). This completes the proof.

This result globalizes straightforwardly to the case where the threefold $\mathscr{W}$ is replaced by a configuration the members of which are almost Fano threefolds.

Theorem 2. Let $[\mathscr{W} \| \mathscr{E}]$ be a configuration the members of which are almost Fano threefolds. Let $\left(\mathscr{A}, \mathscr{B}_{2}, \ldots, \mathscr{B}_{n}\right)$ be line bundles over $\mathscr{W}$, each generated by its global sections, and such that

$$
\mathscr{L}=\mathscr{A} \otimes \mathscr{B}_{2} \otimes \cdots \otimes \mathscr{B}_{n}
$$

has the property that $\left.\mathscr{L}\right|_{\mathscr{M}}=K^{*}{ }_{\mathscr{M}}$ for each member $\mathscr{M}$ of $[\mathscr{W} \| \mathscr{E}]$. Then, the holomorphic family of the configuration

$$
\left[\mathscr{W} \times \mathbb{P}^{n} \| \mathscr{E},\left(\mathscr{A} \otimes h^{2}\right),\left(\mathscr{B}_{2} \otimes h\right), \ldots,\left(\mathscr{B}_{n} \otimes h\right)\right]
$$

contracts by small resolutions to the double covering family of the holomorphic family of the configuration $[\mathscr{W} \| \mathscr{E}]$ induced by the bundle $\mathscr{L}^{2}$ and its space of global sections.

\section{Connected Deformation Space}

Let $\Gamma$ be the directed graph defined as follows: each vertex of $\Gamma$ stands for a configuration, $[\mathscr{W} \| \mathscr{E}]$, where $\mathscr{W}=\mathbb{P}_{1}^{n_{1}} \times \cdots \times \mathbb{P}_{m}^{n_{m}}$ and the tensor product of the line bundles in $\mathscr{E}$ equals the anti-canonical bundle of $\mathscr{W}$. Each directed edge of $\Gamma$ stands for an ordered pair of configurations such that the first contracts to the second by small resolutions.

Lemma 2. $\Gamma$ is connected. 
Proof. Given any configuration, one can split it iteratively until one arrives at a configuration for which each constraint has total degree two and is bilinear in the homogeneous coordinates of two distinct $\mathbb{P}^{n} \mathrm{~s}$, at least one of which is a $\mathbb{P}^{1}$. One can then contract iteratively all the $\mathbb{P}^{n}$ factors for which $n>1$ and then contract $\mathbb{P}^{1} \mathrm{~s}$ as many times as possible. The result is evidently

$$
\left[\begin{array}{l||l}
1 & 2 \\
1 & 2 \\
1 & 2 \\
1 & 2
\end{array}\right]
$$

to which every configuration can therefore be connected.

We can embed $\Gamma$ in a larger directed graph $\Omega$ with a vertex for each configuration satisfying Eq. (3.2), i.e., the members of which are either Calabi-Yau or almost Fano threefolds. The edges of $\Omega$, include, in addition to those of $\Gamma$, an edge for each contraction between almost Fano configurations and an edge for each contraction as in Theorem 2 .

Theorem 3. $\Omega$ is connected.

Proof. Immediate from Lemma 2 and Theorem 2.

Remark. To illustrate the construction in the proof of Lemma 2, we present a path in $\Gamma$ connecting the configuration in Proposition 3 to the one in the proof:

$$
\begin{aligned}
& {\left[\begin{array}{l|l}
3 & 4 \\
1 & 2
\end{array}\right]_{-168}^{2} \leftarrow\left[\begin{array}{l|ll}
3 & 3 & 1 \\
1 & 1 & 1 \\
1 & 0 & 2
\end{array}\right]_{-96}^{3} \leftarrow\left[\begin{array}{l||lll}
3 & 2 & 1 & 1 \\
1 & 1 & 1 & 0 \\
1 & 0 & 1 & 1 \\
1 & 0 & 0 & 2
\end{array}\right]_{-64}^{4} \leftarrow\left[\begin{array}{l|llll}
3 & 1 & 1 & 1 & 1 \\
1 & 1 & 1 & 0 & 0 \\
1 & 0 & 1 & 1 & 0 \\
1 & 0 & 0 & 1 & 1 \\
1 & 0 & 0 & 0 & 2
\end{array}\right]_{-56}^{5} \rightarrow} \\
& \rightarrow\left[\begin{array}{l||l|l}
1 & 2 \\
1 & 2 \\
1 & 2 \\
1 & 2
\end{array}\right]_{-128}^{4} .
\end{aligned}
$$

We have indicated the Euler characteristic and the second Betti number of a manifold belonging to a configuration by the subscripts and superscripts respectively.

Those vertices of $\Omega$ which correspond to AFCI configurations should be understood as representing induced double covering families of Calabi-Yau threefolds. It is a consequence of Proposition 1 that contractions of AFCI configurations induce contractions by small resolutions of the corresponding induced Calabi-Yau double covering families.

\section{Implications for Physics Application}

4-dimensional Minkowski superstring models can be constructed such that some of the internal degrees of freedom of the superstring span a Calabi-Yau manifold 
$\mathscr{M}$. The number of superfields which are massless in the effective 4-dimensional model are determined by the cohomology of the 'internal' manifold $\mathscr{M}$, while the details of the interactions of these superfields depend on the more detailed geometry of $\mathscr{M}$. Inevitably, such superstring models depend on the moduli of $\mathscr{M}$.

With this in mind, Corollary 1 suggests that a superstring model with an internal generic Calabi-Yau manifold $\mathscr{M}^{b} \in\left[\mathscr{W} \| \mathscr{F}_{K}\right]$ may be viewed as a deformation of a superstring model with the (possibly singular) internal Calabi-Yau threefold $\pi(\mathscr{M})$ defined in Eq. (3.5), which in turn was obtained from the one utilizing $\mathscr{M}$ in Eq. (3.3). Similar remarks hold for superstring model interpretation of Theorem 2.

Typically, $\mathscr{M}, \pi(\mathscr{M})$ and $\mathscr{M}^{b}$ have different cohomology and consequently, the spectrum of massless modes in the respective models differs ${ }^{3}$. Note that for $\phi_{K}(w)$ a generic section of $\mathscr{F}_{K}, \Delta+\varepsilon \phi_{K}=0$ with $\varepsilon \in \mathbb{C}$ defines a smooth Calabi-Yau manifold $\mathscr{M}^{b}$, which degenerates 'in the middle' $\varepsilon=0$ into $\pi(\mathscr{M})$. Generally, the transitions suggested by this note would occur at certain (singular) strata of moduli spaces obtained from Eq. (3.1) of the Calabi-Yau threefolds.

It appears very natural to envision a universal moduli space in which the connected moduli spaces of Theorem 3 are embedded; in fact Reid conjectures the existence of a moduli space for threefolds with trivial canonical bundle, the most generic with $b_{2}=0$, in which moduli spaces of Calabi-Yau manifolds occur as singular strata. The present results manifestly resonate strongly with this conjecture. The implication of this to superstring models with internal Calabi-Yau threefolds is self-evident.

We wish to remark on the apparent resemblance of the present results (or Reid's conjecture if proven) and the situation in supersymmetric grand unified models, where several ground states, each with a distinct symmetry, are degenerate and the physical system may tunnel from one ground state to another. There, however, the 'universal moduli space' was known and explicitly constructed (the Higgs parameter space [9]) and one knows of a principle which, at finite temperature and with gravitational effects included, chooses among the ground states. Clearly, superstring counterparts of these features are yet to be found.

\section{References}

1. Hübsch T.: Calabi-Yau Manifolds-Motivations and Constructions, Commun. Math. Phys. 108, 291 (1987). Manifold compactification of superstrings. in: Superstrings, unified theories and cosmology. Furlan G., Jengo R., Pati J.C., Sciama D.W., Sezgin E., Shafi Q., (eds.) (Singapore: World Scientific, 1987; Green P., Hübsch T.: Calabi-Yau manifolds as complete intersections in products of complex projective spaces. Commun. Math. Phys. 109, 99 (1987). Polynomial deformations and cohomology of Calabi-Yau manifolds. Commun. Math. Phys. 113, 505 (1987), Calabi-Yau hypersurfaces in products of semi-ample surfaces. Commun. Math. Phys. 115, 231(1988). Green P., Lütken C.A., Hübsch T.: All the hodge numbers of all complete intersection Calabi-Yau manifolds.

\footnotetext{
${ }^{3}$ As with orbifolds, the nodes of $\pi(\mathscr{M})$ may be innocuous for the string dynamics - in fact, it would be interesting to verify this. It is however not a crucial fact for the interpretation of our present results, since from the physics point of view, these are phase transitions
} 
University of Texas report UTTG-29-87; Candelas P., Lütken C.A., Schimmrigk R.: Complete intersection Calabi-Yau manifolds II. University of Texas report UTTG-11-87

2. Candelas P., Dale A.M., Lütken C.A., Schimmrigk R.: Complete intersection Calabi-Yau manifolds. University of Texas report UTTG-10-87

3. Clemens C.H.: Double solids, Adv. Math. 47, 107 (1983)

4. Reid M.: The moduli space of 3-folds with $K=0$ may nevertheless be irreducible Math. Ann. 278, 329 (1987)

5. Atiyah M.F.: On analytic surfaces with double points. Proc. Roy. Soc. A247, 237 (1958)

6. Hirzebruch F., Werner J.: Some examples of Threefolds with Trivial Canonical Class. Max. Planck Institut für Mathematik (Bonn) Report SFB/MPI 85-58

7. Grauert H.: Über Modifikationen und exzeptionelle analytische Mengen. Math. Ann. 146, 331 (1962); Artin: M. Algebraization of formal moduli. II. Existence of modifications. Ann. Math. 91, 88 (1970)

8. Green P.S., Hübsch: T. Determinantal singularities on Fourfolds and Discriminantal Singularities on Threefolds. (to appear)

9. Reifeartaigh: L.O.: Group structure of gauge theories. Cambridge: Cambridge Press 1986, esp. Chap. 8 and 12 and references therein. For some sample computations see, e.g.,: Kim J.S.: Nucl. Phys. B196, 285 (1982); Abud, M. Anastaze, G. Eckert P., Ruegg H.: Ann. Phys. (NY) 162, 155 (1985); Kaymakcalan, Ö., Michel L., Wali K., McGlinn W.D., Reifeartaigh: L.O.: Nucl. Phys. B267, 203 (1986); Cummings C.J., King R.C.: J. Phys. A19, 161 (1986)

Communicated by S.-T. Yau

Received March 25, 1988; in revised form June 6, 1988 
